\title{
31. Commutativity of Some Continuous Magnitude
}

\author{
By Kiyoshi IsÉkI and Hajime SugItA \\ (Comm. by K. KUnUGI, M.J.A., March 12, 1960)
}

A magnitude $M$ is a set of elements $a, b, c, \cdots$ and a binary operation called sum satisfying the following Conditions I-IV.

I. For every pair $a, b$ of $M$, the sum $a+b$ exists.

II. For every $a, b, c,(a+b)+c=a+(b+c)$.

Definition. If $a, b \in M$, then $a<b$ means that there is an element $x$ such that $a+x=b . \quad a>b$ means $b<a$.

III. $a, b<a+b$ for every $a, b$.

IV. If $a, b$ are distinct, then either $a<b$ or $b<a$.

Therefore, any magnitude $M$ is linear ordered set.

A magnitude $M$ is Archimedean, if $a>b$, then there is a positive integer $n$ such that $n b>a$.

Since O. Hölder, some mathematicians, R. Baer, H. Cartan, F. Loonstra and F. A. Behrend [1], have proved that any Archimedean magnitude is commutative: $a+b=b+a$ for every element $a, b$ (see H. G. Forder [2]).

A magnitude $M$ is continuous, if every bounded subset has the least upper bound.

In this Note, we shall prove the following

Theorem. Any continuous magnitude is commutative.

To prove it, we shall show that a continuous magnitude is Archimedean.

Remark. For the proof, Condition III is essential.

Proof. Suppose that $M$ is not Archimedean, then there are two elements $a, b$ such that $a<b$ and $n a \leq b$ for $n=1,2, \cdots$.

Consider the set of elements $n a(n=1,2, \cdots)$, then the set is bounded, and has a least upper bound $c$ such that $n a \leq c$ for all $n=1,2, \cdots$ and for $c^{\prime}<c$, there is an integer $m$ such that $m a>c^{\prime}$. From $a<c$, there is an element $x$ such that $a+x=c$. By Condition II, we have $x<a+x=c$. Therefore there is an integer $m$ such that $m a>x$. Hence we have $a+m a>a+x$. (This is proved without commutative law.) This shows $(m+1) a>c$, which is a contradiction. Therefore we complete the proof.

\section{References}

[1] F. A. Behrend: A system of independent axioms for magnitudes, Journal and Proceedings of Royal Society of New South Wales, 87, 27-30 (1953).

[2] H. G. Forder: The Foundations of Euclidean Geometry, New York (1927). 